\title{
The effect of \\ ursodeoxycholic acid on Messenger RNA hepcidin expression associated with liver fibrosis in perposed cholestasis jaundice
}

Objectives: Cholestasis in extra hepatic obstruction induce cells stress and associated with the induction of inflammatory that correlate with hepcidin Messenger RNA (mRNA) expression. In our research we tried to analize the correlation between induced chlolestatis with MRNA expression of hepcidin.

Methods : By using the 24 of Wistar rats - male with an average weight of standard $240 \mathrm{~g}, 200 \mathrm{~g}$, were divided into 2 groups; artificial cholestasis without intervention and Artificial cholestasis with intervention of ursodeoxycholic acid. We use total RNA isolation from rat liver tissue by using GeneJet RNA Purification Kit (Thermo Sciencetific).

Results : RNA levels were measured using a TECAN Infinate 200 NanoQuant. Hepcidin mRNA expression was measured by real-time Quantitive recieve transcription PCR LightCycler.

Conclusion : MRNA expression of hepcidin decreased significantly $(p<0.01)$ in the ursodeoxycholic acid group. Increased Plasma hepcidin is proven associated with progression of liver fibrosis and correlated with prognosis.

KEYWORDS: mRNA hepcidin, cholestasis, liver fibrosis

\section{Introduction}

Cholestasis is defined as a state with increased levels of serum bilirubin direk more than $20 \%$ of total bilirubin. In neonates itself abnormal bilirubin can occur at 14 days of life. This cholestasis can be caused by abnormalities of intrahepatic or post-hepatic anatomic abnormality that causes obstruction $[1,2]$.

Molecular pathways associated with chronic inflammation of the liver that causes fibrosis of the liver was investigated further. Current treatment is to focus on the etiology and to eliminate oxidative stress, reduce hepatic stellate cell activation that reduces myofibroblast proliferation and increasing degradation of the extracellular matrix [3,4].

Anti-fibrotic liver that is often used is ursodeoxycholic acid as a single treatment or in combination with corticosteroids has proven itself as the leading therapy in some previous studies. The working principle of ursodeoxycholic acid is not directly reduce the inflammation of the liver, but by eliminating the etiology by interrupting immune-mediated pathways $[4,5]$.

Cholestasis can reduce Hepcidin mRNA expression and significantly down regulation of hepatic hepcidin mRNA expression. The state of cholestasis in various previous studies in animals and humans raises the stress associated with the induction of inflammatory cytokines and IL-6 [6-9].

Hepcidin in humans was first isolated and characterized as highly disulfide-bonded peptides with activity of anti-microbial. In theory hepcidin will be regulated when there is inflammation in the liver or chronic infections, or begin fibrosis example in the case of biliary atresia. Hepcidin mRNA expression can be done through qRT-PCR, or ELISA assay of plasma hepcidin. Increased Plasma hepcidin is proven associated with progression of liver fibrosis and correlated with prognosis [10-13].

\section{Methods}

By using the 24 of Wistar rats - male with an average weight of standard $240 \mathrm{gr}, 200 \mathrm{gr}$, were divided into three groups, care and use of rat

\section{Bilommi $\mathrm{R}^{1 *} \&$ Sandra $\mathrm{Y}^{2}$}

${ }^{1}$ Department of Pediatrics Surgery, Mitra Keluarga Hosital, Jakarta, Indonesia

${ }^{2}$ Department of Biochemistry and Molucular Genetics, YARSI Medical Faculty, Jakarta, Indonesia

*Author for correspondence:

rbilommi@gmail.com 
strictly follow the protocol approved by the Ethics Committee YARSI. The 24 Mice received laparotomy and had extra hepatic duct ligation, they were then randomly allocated into three groups.

-First group (artificial cholestasis without intervention then after 4 weeks we take the tissues of the hepar)

-Second group after 2 weeks of artificial cholestasis without intervention they were given ursodeoxycholic acid as an injection of a single dose (dose as the same as human correlates with the weight) then after 2 weeks we take the tissues of the hepar).

\section{Results}

We use total RNA isolation from rat liver tissue by using GeneJet RNA Purification Kit (Thermo Sciencetific). RNA levels were measured using a TECAN Infinate 200 NanoQuant. Hepcidin mRNA expression was measured by real-time Quantitive recieve transcription PCR LightCycler. 480 Real Time PCR System. QCT Real Time PCR was performed using LightCycler RNA Master SYBR Green (ROCHE) with the following protocols: 1 cycle at a temperature of $61^{\circ} \mathrm{C}$ for 20 minutes to a reverse transcrition, 1 cycle for 30 seconds at $95^{\circ} \mathrm{C}$ for the initiation of denaturation, 45 cycles at $96,60.72^{\circ} \mathrm{C}$ for $1,5,25$ seconds for amplification, one cycle at $95,65,95^{\circ} \mathrm{C}$ for $0,15,0$ seconds in the melting curve, 1 cycle at $40^{\circ} \mathrm{C}$ for 30 seconds for cooling. B actin selected as housekeeping gene and the relative expression of hepcidin is calculated by comparing the value of $\mathrm{c}(\mathrm{T})$ hepcidin terhadapa B actin using 2 -ddc formula (T). Sequence primers used for hepcidin, forwardCACAGCAGAACAGAAGGCATG 5'-3', 5'-CTTCTGCTGTAAATGCTGT reverse-3; $B$ actin-ACCACAGCTGAGGGAAATC forward 5'-3'; reverse 5'-AGAGGTCTTACGGATGTCAACG-3

MRNA expression of hepcidin decreased significantly $(\mathrm{p}<0.01)$ in the intervention group.

\section{Discussion}

Jaundice results from the retention of bile. Bile formation is a complex process and is readily disrupted by a variety of hepatic insults. Thus, jaundice, a yellow discoloration of skin and sclerae (icterus), occurs when systemic retention of bilirubin produces serum levels above 2.0 $\mathrm{mg} / \mathrm{dL}$ (the normal level in adults is below 1.2 $\mathrm{mg} / \mathrm{dL}$ ). Cholestatic jaundice, characterized by elevation of serum conjugated bilirubin, is an uncommon but potentially serious condition that indicates hepatobiliary dysfunction. Cholestatic jaundice affects approximately 1 in every 2,500 infants. The most common causes of cholestatic jaundice in the first months of life are biliary atresia and neonatal hepatitis, which account for most cases. Neonatal hepatitis has referred to a histologic appearance of widespread giant cell transformation. Although giant cell transformation is recognized to be nonspecific and may be associated with infectious, metabolic, and syndromic disorders, this term is used to be consistent with the older literature reviewed for this guideline. The remaining cases are caused by a variety of other disorders, including extrahepatic obstruction from common duct gallstone or choledochal cyst; metabolic disorders such as tyrosinemia, galactosemia, and hypothyroidism; inborn errors of bile acid metabolism; Alagille syndrome; infection; and other rare disorders $[1,4,11,16,17]$.

Obstructive jaundice (OJ) increases the risk of liver injury and sepsis, leading to increased mortality [6,7,11-17].

In our research mRNA expression of hepcidin decreased significantly $(p<0.01)$ in the intervention group.

Endotoxin-induced cholestasis weakens Hepcidin Expression. Endotoxin is a potent inducer of inflammatory cytokines, including IL-6, and is known to induce the expression of hepcidin. Our findings indicate that the hepcidin, a natural antimicrobial peptide, significantly suppressed in mice yellow when extra hepatic duct ligation. Endotoxins can stimulate the expression of inflammatory cytokines, including tumor necrosis factor alpha (TNF- $\alpha$ ), IL-1 and IL-i.e. 6:31 Nemeth et al have shown that hepcidin expression is induced only by IL-6, but not by IL-1 or TNF - in humans. In contrast, Lee et al have shown that hepcidin transcription in hepatocytes stimulated not only by the murine IL-6, but also by IL-1 i.e., reflecting the fact that there are differences between species in the regulation of hepcidin expression by inflammatory cytokines. IL- 6 has been identified as essential for homeostasis during inflammation, due to animal IL-6-deficient are more susceptible to the hepatotoxic challenges, such as endotoxin.34, 35 IL-6 is also a positive inducer of hepatocyte proliferation.36 Signal transduction through gp130 divided among subfamily of receptors for the cytokine IL- 6 are associated. Interestingly, mice chronic cholestasis without gp130 
expression results more bacterial infections and mortality are higher, which confirms the importance of gp130 in innate immunity and IL-6 signaling.37 intrahepatic Stat3 activation predictable outcome, 38 and STAT3 knockout mice showed significantly higher death of widths after cecal ligation and puncture sepsis. Kano et al. have shown that STAT3 signaling in the endothelium have anti-inflammatory as an important mediator of the endotoxin-induced inflammation $[11,12]$.

In the study by Graf et al, hydrophobic bile acids compromise IL-6 signaling through both downregulation of gp130 and p38MAPK dependent inhibition of STAT3 phosphorylation. In this study, a significant decrease of hepcidin, gp130 mRNA, protein expression and protein pSTAT3 pSTAT3 nuclear translocation in patients with cholestatic liver and yellow mice compared with noncholestatic patients and sham-operated rats.
Hydrophobic bile acids can reduce hepcidin expression in response to IL-6 stimulation by inhibiting STAT3 phosphorylation in hepatocytes. Hepcidin has significant antimicrobial, especially for E.coli. In the study Huang et al Hepcidin is a cysteine-rich molecule containing four disulfide bonds and can also inhibit the growth of Salmonella typhimurium and Mycobacterium tuberculosis. A decrease of hepcidin in the final stages of biliary atresia showed a loss of protection, which could explain the progressive decline of the patient.

\section{Conclusion}

The presentation of liver fibrosis due to cholestatis jaundice can me measured by hepcidin mRNA expression. Increased Plasma hepcidin is proven associated with progression of liver fibrosis and correlated with prognosis. In our research Expression of hepcidin due to cholestasis proven decreased by administering of drugs ursodeoxycholic Acid.

\section{REFERENCES}

1. A-Kader HH, Balistreri WF. Cholestasis (2003). In: WB Saunders (ed.), Nelson Textbook of Pediatrics, 17 th edn.

2. Alagille D, Estrada A, Hadchouel M, et al. Syndromic paucity of interlobular bile ducts (Alagille syndrome or arteriohepatic dysplasia): review of 80 cases. J. Pediatr. 110,195-200(1987).

3. Altman RP, Buchmiller TL. The jaundiced infant: biliary atresia. (2005).

4. Pediatric Surgery, 6th edn. pp 1603-1619. Mosby (2006).

5. Czaja AJ. Hepatic inflammation and progressive liver fibrosis in chronic liver disease. World J. Gastroenterol. 20(10),25152532(2014).

6. EASL Clinical Practice Guidelines: Management of Cholestatic Liver Diseases. J. Hepatology 51,237-267(2009).

7. Huang $\mathrm{YH}$, Hui $\mathrm{YH}$, Huang CC, et al. Liver Hepcidin and Stainable Iron Expression In
Biliary Atresia. Pediatric Research. 59(5)(2006).

8. Chuang JH, Lee SY, Chen WJ, et al. Changes in bacterial concentration in the liver correlate with that in the hepaticojejunostomy after bile duct reconstruction: implication in the pathogenesis of postoperative cholangitis. World J. Surg. 25(12),1512-1518 (2001).

9. Park $\mathrm{CH}$, Valore EV, Waring AJ, et al. Hepcidin, a urinary antimicrobial peptide synthesized in the liver. J. Biol. Chem. 276(11),7806-7810 (2001).

10. Huang $\mathrm{YH}$, Yu-Hsieh $\mathrm{H}$, Huang CC, et al. Liver hepcidin and stainable iron expression in biliary atresia. Pediatr. Res. 59(5),662-666 (2006).

11. Aoki CA, Rossaro L, Ramsamooj R, et al. Liver hepcidin mRNA correlates with iron stores, but not inflammation, in patients with chronic hepatitis C. J. Clin. Gastroenterol. 39(1),71-74 (2005).

12. Bergmann OM, Mathahs MM, Broadhurst $\mathrm{KA}$, et al. Altered expression of iron regulatory genes in cirrhotic human livers: clues to the cause of hemosiderosis? $L a b$. Invest. 88,1349-1357 (2008).

13. Jaroszewicz J, Rogalska M, Flisiak R. Serum prohepcidin reflects the degree of liver function impairment in liver cirrhosis. Biomarkers. 13(5),478-485(2008).

14. Pietrangelo A, Dierssen U, Valli $L$, et al. STAT3 is required for IL-6-gp130dependent activation of hepcidin in vivo. Gastroenterology.132(1),294-300(2007).

15. Choi SO, Cho YS, Kim HL, Park JW. ROS mediate the hypoxic repression of the hepcidin gene by inhibiting C/EBPalpha and STAT-3. Biochem. Biophys. Res. Commun. 356(1),312-317(2007).

16. Graf D, Kohlmann C, Haselow K, et al. Bile acids inhibit interleukin- 6 signaling via gp130 receptor-dependent and -independent pathways in rat liver. Hepatology. 44,12061217(2006).

17. Kaplan MM, Gershwin ME. Primary biliary cirrhosis. N. Engl. J. Med. 353(12),12611273(2005). 